BULl. AUSTRAL. MATH. SOC.

VOL. 30 (1984), 37-43.

\title{
ON P-VALENT STARLIKE FUNCTIONS WITH REFERENCE TO THE BERNARDI INTEGRAL OPERATOR
}

\author{
VINOD KUMAR AND S.L. SHUKLA
}

Let $S_{p}^{*}(A, B)$ denote the class of certain $p$-valent starlike functions. Recently G. Lakshma Reddy and K.S. Padmanabhan [Bull. Austral. Math. Soc. 25(1982), 387-396] have shown that the function $g$ defined by

$$
g(z)=(c+p) z^{-c} \int_{0}^{z} t^{c-1} f(t) d t, c=1,2,3, \ldots,
$$

belongs to the class $S_{p}^{*}(A, B)$ if $f \in S_{p}^{*}(A, B)$. The technique used by them fails when $c$ is any positive real number. In this paper, by employing a more powerful technique, we improve their result to the case when $c$ is any real number such that $c \geq-p(1+A) /(1+B)$.

\section{Introduction}

Let $S_{p}^{*}(A, B)$ denote the class of functions of the form

$$
f(z)=a_{p} z^{p}+\sum_{n=p+1}^{\infty} a_{n} z^{n}, p \geq 1,
$$

which are analytic in the unit disc $E=\{z:|z|<1\}$, and satisfy $(1.2)$

$$
z \frac{f^{\prime}(z)}{f(z)}=p \frac{1+A w(z)}{1+B w(z)}, z \in E,
$$

where $-1 \leq A<B \leq 1, w$ in analytic in $E$, and satisfies $w(0)=0$

Received 20 January 1984

Copyright Clearance Centre, Inc. Serial-fee code: 0004-9727/84 $\$ A 2.00+0.00$ 
and $|w(z)|<1$ for $z \in E$. Evidently, the functions in $S_{p}^{\star}(A, B)$ are p-valent starlike in $\mathbf{E}$.

Bernardi [1] has shown that, if the function $f$ is univalent starlike in $E$, then so is the function $g$ given by

$$
g(z)=(c+1) z^{-c} \int_{0}^{z} t^{c-1} f(t) d t
$$

where $c$ is a positive integer. This result has been improved by Miller et al. [5, Theorem 2] to the case when $c$ is a positive real number. Recently, Reddy and Padmanabhan [6, Theorem 1] have extended the result of Bernardi [1] by proving that, if $f \in s_{p}^{*}(A, B)$, then so does the function $g$ given by

$$
g(z)=(c+p) z^{-c} \int_{0}^{z} t^{c-1} f(t) d t
$$

where $c$ is a positive integer. The classical technique used by Reddy and Padmanabhan [6] fails when $c$ is any positive real number. It is therefore natural to ask whether their result can be improved for real $c$.

The object of the present paper is to establish a theorem which improves, in particular, the result of Reddy and Padmanabhan [6, Theorem 1] to the case when $c$ is a real number such that $c \geq-p(1+A) /(1+B)$. It is worth noting that the technique employed to prove our theorem is different from those used by Miller et al. [5] and Reddy and Padmanabhan [6]. In fact our important tool is Lemma 2.1 , to be proved in section 2 , which provides a geometrical definition of the class $S_{p}^{*}(A, B)$.

\section{Preliminary lemmas}

To establish our main result we require the following lemmas:

LEMMA 2.1. A function $f$ of the form (1.1) belongs to $S_{p}^{*}(A, B)$, $-1 \leq A<B<1$, if and only if

$$
\left|z \frac{f^{\prime}(z)}{f(z)}-m\right|<M, \quad z \in E,
$$

where

$$
m=p(1-A B) /\left(1-B^{2}\right) \text { and } M=p(B-A) /\left(1-B^{2}\right) .
$$


Proof. Let $f \in S_{p}^{\star}(A, B)$. Then from $(1.2)$ we have

$$
\begin{aligned}
z \frac{f^{\prime}(z)}{f(z)}-m & =\frac{(p-m)+(A p-B m) w(z)}{1+B w(z)} \\
& =M h(z)
\end{aligned}
$$

where $h(z)=-(B+w(z)) /(1+B w(z))$. Since $|h(z)|<1$, the inequality $(2.1)$ follows from $(2.3)$.

$$
\begin{aligned}
& \text { Conversely, let } \mathrm{f} \text { satisfy (2.1). Then } \\
& || z \frac{\mathrm{f}^{\prime}(\mathrm{z})}{\mathrm{Mf}(\mathrm{z})}-\frac{\mathrm{m}}{\mathrm{M}} \mid<1, \quad \mathrm{z} \in \mathrm{E} .
\end{aligned}
$$

Let

$$
q(z)=z \frac{f^{\prime}(z)}{M f(z)}-\frac{m}{M}
$$

and we define

$$
w(z)=\frac{q(0)-q(z)}{1-q(0) q(z)} .
$$

Clearly the function $w$ is analytic in $E$, and satisfies $w(0)=0$ and $|w(z)|<1$ for $z \in E$. Since $q(0)=-B$, from (2.5) we get

$$
q(z)=-(B+w(z)) /(1+B w(z)) \text {. }
$$

Eliminating $q(z)$ from (2.4) and (2.6) we get (1.2). Hence $f \in S_{p}^{*}(A, B)$. Note: (i) The condition (2.1) can be written in the form

$$
\left|\frac{\left(z \mathrm{f}^{\prime}(z) / \mathrm{f}(\mathrm{z})\right)-(\mathrm{p}(1+\mathrm{A}) /(1+\mathrm{B}))}{\mathrm{p}-(\mathrm{p}(1+\mathrm{A}) /(1+\mathrm{B}))}-\frac{1}{1-\mathrm{B}}\right|<\frac{1}{1-\mathrm{B}}, z \in E .
$$

Now as $B \rightarrow 1$ and $A=-(1-2 \beta), 0 \leq \beta<1$, this inequality reduces to $\operatorname{Re}\left\{z f^{\prime}(z) / f(z)\right\}>p B, z \in E$, which is precisely a necessary and sufficient condition for $f \in \mathrm{s}_{\mathrm{p}}^{*}(2 \beta-1,1)$. Thus including the limiting case $B \rightarrow 1$, the results proved with the help of Lemma 2.1 will hold for $-1 \leq A<B \leq 1$.

(ii) Throughout this paper $m$ and $M$ are given by (2.2).

LEMMA 2.2. If the fronction $w$ is analytic for $|z| \leq r<1$, $w(0)=0$ and $\left|w\left(z_{0}\right)\right|=\max _{|z|=x}|w(z)|$, then $z_{0} \omega^{\prime}\left(z_{0}\right)=k w\left(z_{0}\right)$, where $k$ is a real number such that $k \geq 1$. 
The above lemma is due to Jack [3].

\section{Main result}

THEOREM. If $f \in S_{p}^{*}(A, B)$ and $g$ is defined by

$$
g(z)=\left((c+p \alpha) z^{-c} \int_{0}^{z} t^{c-1} f^{\alpha}(t) d t\right)^{1 / \alpha}
$$

where $\alpha$ and $c$ are real numbers such that $\alpha>0$ and $c \geq-p a(1+A) /(1+B)$. Then the function $g$ also belongs to $S_{p}^{*}(A, B)$.

In (3.1) powers denote principal ones.

Proof. Let us define a function $w$ such that

$$
w(z)=\frac{z g^{\prime}(z) / g(z)-p}{A p-B z g^{\prime}(z) / g(z)}
$$

so that

$$
z \frac{g^{\prime}(z)}{g(z)}=p \frac{1+A w(z)}{1+B w(z)}
$$

where $w$ is either analytic or meromorphic in E. Clearly $w(0)=0$. We claim that $w$ is analytic in $E$, and $|w(z)|<1$ for $z \in E$, which we will prove by contradiction.

$$
\text { From (3.1) and (3.2) we have }
$$

$$
(c+p \alpha)\left\{\frac{f(z)}{g(z)}\right\}^{\alpha}=\frac{(c+p \alpha)+(A p \alpha+B c) w(z)}{1+B w(z)} .
$$

Logarithmic differentiation of (3.3) yields

$$
z \frac{f^{\prime}(z)}{f(z)}-m=\frac{(p-m)+(A p-B m) w(z)}{1+B w(z)}-\frac{p(B-A) z w^{\prime}(z)}{\{1+B w(z)\}\{(c+p \alpha)+(A p \alpha+B c) w(z)\}} .
$$

Let $r^{*}$ be the distance, from the origin, of the pole of $w$ nearest the origin. Then $w$ is analytic in $|z|<r_{0}=\min \left\{r^{*}, 1\right\}$. By Lemma 2.2, for $|z| \leq r\left(r \leq r_{0}\right)$, there exists a point $z_{0}$ such that

$$
z_{0} w^{\prime}\left(z_{0}\right)=k w\left(z_{0}\right), k \geq 1 \text {. }
$$

From (3.4) and (3.5) we have

$$
z_{0} \frac{f^{\prime}\left(z_{0}\right)}{f\left(z_{0}\right)}-m=\frac{N\left(z_{0}\right)}{D\left(z_{0}\right)}
$$

where 


$$
\begin{aligned}
N\left(z_{0}\right)= & (p-m)(c+p \alpha)+\{(c+p \alpha)(A p-B m)+(A p \alpha+B c)(p-m)-k p(B-A)\}_{w}\left(z_{0}\right) \\
& +\{(A p \alpha+B c)(A p-B m)\} w^{2}\left(z_{0}\right)
\end{aligned}
$$

and

$$
D\left(z_{0}\right)=(c+p \alpha)+(A p \alpha+2 B c+B p \alpha) w\left(z_{0}\right)+B(A p \alpha+B c) w^{2}\left(z_{0}\right) .
$$

Now suppose that it were possible to have $\max _{|z|=r}|w(z)|=\left|w\left(z_{0}\right)\right|=1$ for some $r, r<r_{0} \leq 1$. Then by using the identities Ap $-B m=-M$ and $B-A=\left(M^{2}-(m-p)^{2}\right) /(M p)$, we have

$$
\left|N\left(z_{0}\right)\right|^{2}-M^{2}\left|D\left(z_{0}\right)\right|^{2}=a+2 b \operatorname{Re}\left\{w\left(z_{0}\right)\right\}
$$

where

$$
a=k p(B-A)\{k p(B-A)+2 M(C+p \alpha)+2 M B(A p \alpha+B C)\}
$$

and

$$
b=k p(B-A) M\{(A p \alpha+B C)+B(c+p \alpha)\} .
$$

From (3.7) we have

$$
\left|\mathrm{N}\left(\mathrm{z}_{0}\right)\right|^{2}-\mathrm{M}^{2}\left|\mathrm{D}\left(\mathrm{z}_{0}\right)\right|^{2}>0
$$

provided $a \pm 2 b>0$. Now

$$
\begin{aligned}
a+2 b & =k p(B-A)[k p(B-A)+2 M(1+B)\{c(1+B)+p \alpha(1+A)\}] \\
& >0, \text { provided c } 2-p \alpha(1+A) /(1+B),
\end{aligned}
$$

and

$$
\begin{aligned}
a-2 b & =k p(B-A)[k p(B-A)+2 M(1-B)\{c(1-B)+p \alpha(1-A)\}] \\
& >0, \text { provided } c \geq-p \alpha(1-A) /(1-B) .
\end{aligned}
$$

Thus from (3.6) and (3.8) it follows that

$$
\left|z_{0} \frac{f^{\prime}\left(z_{0}\right)}{f\left(z_{0}\right)}-m\right|>M
$$

provided

$$
\begin{aligned}
c & \geq \max \{-\mathrm{p} \alpha(1+\mathrm{A}) /(1+\mathrm{B}),-\mathrm{p} \alpha(1-\mathrm{A}) /(1-\mathrm{B})\} \\
& =-\mathrm{p} \alpha(1+\mathrm{A}) /(1+\mathrm{B}) .
\end{aligned}
$$

But this is, in view of Lemma 2.1 , contrary to our assumption $f \in \mathrm{S}_{\mathrm{p}}^{*}(\mathrm{~A}, \mathrm{~B})$. Therefore we cannot have $|w(z)|=1$ in $|z|<r_{0}$. Since 
$|w(0)|=0,|w(z)|$ is continuous and $|w(z)| \neq 1$ in $|z|<x_{0}$, w cannot have a pole at $|z|=r_{0}$. Since $r_{0}$ is arbitrary, we conclude that $w$ is analytic in $E$, and satisfies $|w(z)|<1$ for $z \in E$. Hence, from (3.2), $g \in S_{p}^{*}(A, B)$.

Remark. It is evident that, for $\alpha=1$, the above theorem improves the result of Reddy and Padmanabhan [6,Theorem I].

If we set $A=-(1-2 \beta)$, where $0 \leq \beta<1$, and $a_{1}=p=B=1$, the class $S_{p}^{*}(A, B)$ xeduces to the well known class $S^{*}(\beta)$ of univalent starlike functions of order $\beta$. For the class $S^{*}(\beta)$, the undermentioned corollary follows immediately from the above theorem.

COROLLARY. Let $\alpha$ and $c$ be real numbers such that $\alpha>0$ and $c \geq-\alpha \beta$. If $f \in S^{*}(\beta)$, then the function $g$ defined by

$$
g(z)=\left[\frac{c+\alpha}{z^{c}} \int_{0}^{z} t^{c-1} f^{\alpha}(t) d t\right] 1 / \alpha
$$

is also an element of $S^{*}(\beta)$.

Remarks (i) A result of Miller et al. [5, Theorem 2] turns out to be a particular case of the above corollary when $B=0$.

(ii) Gupta and Jain [2,Theorem 1] have also shown that the function $g$ defined by (3.9) belongs to the class $S^{*}(\beta)$ if $f \in S^{*}(\beta)$. However, as an example, the integral operator

$$
g(z)=\left[\frac{11}{4 z^{2}} \int_{0}^{z} t f^{3 / 4}(t) d t\right]^{4 / 3}
$$

can be studied by the above corollary and not by the result of Gupta and Jain, since the technique followed by them fails when at least one of $\alpha$ and $c$ is not a positive integer.

Problem. Very recently, Kumar and Shukla [4,Theorem $1(i)]$ have shown that the function $g$ given by (3.9) belongs to $S^{*}(\beta)$ even when $c$ is a complex number such that $\operatorname{Re}(c) \geq-\alpha \beta$. It would be interesting to show that the function $g$ given by (3.1) belongs to $S_{p}^{*}(A, B)$ when $C$ is a complex number such that $\operatorname{Re}(c) \geq-p \alpha(1+A) /(1+B)$. 


\section{References}

[1] S.D. Bernardi, "Convex and starlike univalent functions", Trans. Amer. Math. Soc. 135 (1969), 429-446.

[2] Ved P. Gupta and Pawan K. Jain, "On starlike functions", Rend. Mat. 9 (1976), 433-437.

[3] I.S. Jack, "Functions starlike and convex of order $\alpha$ ", J. London Math. Soc. (2) 3 (1971), 469-474.

[4] Vinod Kumar and S.L. Shukla, "Bazilevič integral operators", Rend. Mat. 3 (1983).

[5] Sanford S. Miller, Petru T. Mocanu and Maxwell O. Reade, "Starlike integral operators", Pacific J. Math. 79 (1978), 157-168.

[6] G. Lakshma Reddy and K.S. Padmanabhan, "On analytic functions with reference to Bernardi integral operator", Bull. Austral. Math. Soc. 25 (1982), 387-396.

Dr V. Kumar,

Department of Mathematics,

Christ Church College,

Kanpur-208001,

India.

Dr S.L. Shukla,

Department of Mathematics,

Janta College, Bakewar,

Etawah-206124,

India. 